\title{
Identification of a distinct imaging phenotype may improve the management of palindromic rheumatism
}

\author{
Kulveer Mankia, ${ }^{1,2}$ Maria-Antonietta D'Agostino, ${ }^{1,3,4}$ Richard J Wakefield, 1,2 \\ Jackie L Nam, ${ }^{1,2}$ Waqar Mahmood, ${ }^{1}$ Andrew J Grainger, ${ }^{2,5}$ Paul Emery ${ }^{1,2}$
}

\section{Handling editor Josef $S$ Smolen}

- Additional material is published online only. To view please visit the journal online (http://dx.doi.org/10.1136/ annrheumdis-2018-214175)

'Department of Rheumatology, Leeds Institute of Rheumatic and Musculoskeletal Medicine University of Leeds, Leeds, UK 'Department of Rheumatology, NIHR Leeds Biomedical Research Centre, Leeds Teaching Hospitals NHS Trust, Leeds, UK ${ }^{3}$ Department of Rheumatology, APHP, Hopital Ambroise Paré, Paris, France

${ }^{4}$ Department of Rheumatology, INSERM U1173, Laboratoire d'Excellence INFLAMEX, UFR Simone Veil, Versailles-SaintQuentin University, Versailles, France

${ }^{5}$ Department of Radiology Leeds Teaching Hospitals NHS Trust, Leeds, UK

\section{Correspondence to} Professor Paul Emery, Leeds Institute of Rheumatic and Musculoskeletal Medicine, Chapel Allerton Hospital, Leeds LS7 4SA, UK;

p.emery@leeds.ac.uk

Received 24 July 2018 Revised 2 September 2018 Accepted 3 September 2018 Published Online First

8 October 2018

\section{Check for updates}

(C) Author(s) (or their employer(s)) 2019. No commercial re-use. See rights and permissions. Published by BMJ.

To cite: Mankia K

D'Agostino M-A,

Wakefield RJ, et al

Ann Rheum Dis

2019:78:43-50

\section{ABSTRACT}

Objectives To use high-resolution imaging to

characterise palindromic rheumatism (PR) and to

compare the imaging pattern observed to that seen in

new-onset rheumatoid arthritis (NORA).

Methods Ultrasound (US) assessment of synovitis,

tenosynovitis and non-synovial extracapsular

inflammation $(\mathrm{ECl})$ was performed during and between

flares in a prospective treatment-naive PR cohort. MRI

of the flaring region was performed where possible.

For comparison, the same US assessment was also

performed in anticyclic citrullinated peptide (CCP)

positive individuals with musculoskeletal symptoms

(CCP+ at risk) and patients with NORA.

Results Thirty-one of 79 patients with PR recruited were assessed during a flare. A high frequency of $\mathrm{ECI}$ was identified on US; 19/31 (61\%) of patients had ECI including $12 / 19(63 \%)$ in whom ECl was identified in the absence of synovitis. Only 7/31 (23\%) patients with PR had synovitis (greyscale $\geq 1$ and power Doppler $\geq 1$ ) during flare. In the hands/wrists, ECI was more prevalent in PR compared with NORA and CCP+ at risk (65\% vs $29 \%$ vs $6 \%, \mathrm{p}<0.05)$. Furthermore, $\mathrm{ECl}$ without synovitis was specific for PR ( $42 \%$ PR vs $4 \%$ NORA $(p=0.003)$ and $6 \% C C P+$ at risk $(p=0.0012))$. Eleven PR flares were captured by MRI, which was more sensitive than US for synovitis and ECI. 8/31 (26\%) patients with PR developed RA and had a similar US phenotype to NORA at progression.

Conclusion PR has a distinct US pattern characterised by reversible $\mathrm{ECl}$, often without synovitis. In patients presenting with new joint swelling, US may refine management by distinguishing relapsing from persistent arthritis.

Early diagnosis and treatment of inflammatory arthritis (IA) is associated with less joint damage and a higher chance of achieving remission. ${ }^{1}$ However, identifying and treating IA at the earliest opportunity can be challenging as many patients with disease-specific autoantibodies and/or inflammatory joint symptoms do not necessarily develop persistent arthritis. An important example is patients with palindromic rheumatism (PR).

$\mathrm{PR}$ is characterised by intermittent flares of articular and periarticular inflammation. Up to $50 \%$ of patients with PR will eventually develop rheumatoid arthritis (RA), with those that are anticyclic citrullinated peptide (CCP) antibody positive at highest risk of progression. ${ }^{2-4}$ However, the time to progression is variable and many anti-CCP positive patients with PR do not develop persistent arthritis,

\section{Key messages}

What is already known?

- Palindromic rheumatism (PR) is characterised by flares of joint pain and swelling and is associated with anti-CCP antibodies.

- In patients presenting with new joint swelling, PR can be difficult to distinguish from persistent arthritis, despite having a more favourable prognosis.

What does this study add?

- This study has identified a distinct imaging pattern in PR, characterised by extracapsular inflammation, often without synovitis.

How might this impact on future practice?

- Ultrasound may refine the management of PR by distinguishing it from early persistent arthritis.

even after several years of follow-up. ${ }^{5}$ Identifying patients with this favourable prognosis from those with early persistent IA is important; the latter require early disease-modifying therapy, whereas the former can often be monitored with a more conservative approach.

In clinical practice, distinguishing true PR from a new presentation of IA can be challenging; many patients require multiple assessments before a diagnosis is made. ${ }^{6}$ High-resolution imaging, particularly ultrasound (US), is recommended as part of the diagnostic workup for suspected $\mathrm{RA}^{7}$ with many rheumatologists now using US in their routine practice. ${ }^{8}$ Imaging studies in PR have, however, been limited. ${ }^{9}{ }^{10}$ This is likely due to the difficulty in capturing this group of patients and the sporadic nature of flares. We therefore aimed to comprehensively describe the imaging phenotype of PR in a prospective treatment-naive cohort, both during and between flares. We then sought to compare this to the imaging findings in (1) anti-CCP positive individuals with musculoskeletal (MSK) symptoms (CCP+ at risk) and (2) patients with new-onset RA (NORA). We hypothesised that both synovial and non-synovial extracapsular (EC) structures are important disease targets in PR and that imaging would reveal a distinct pattern of inflammation which may be used to distinguish PR from patients presenting with early persistent IA. 


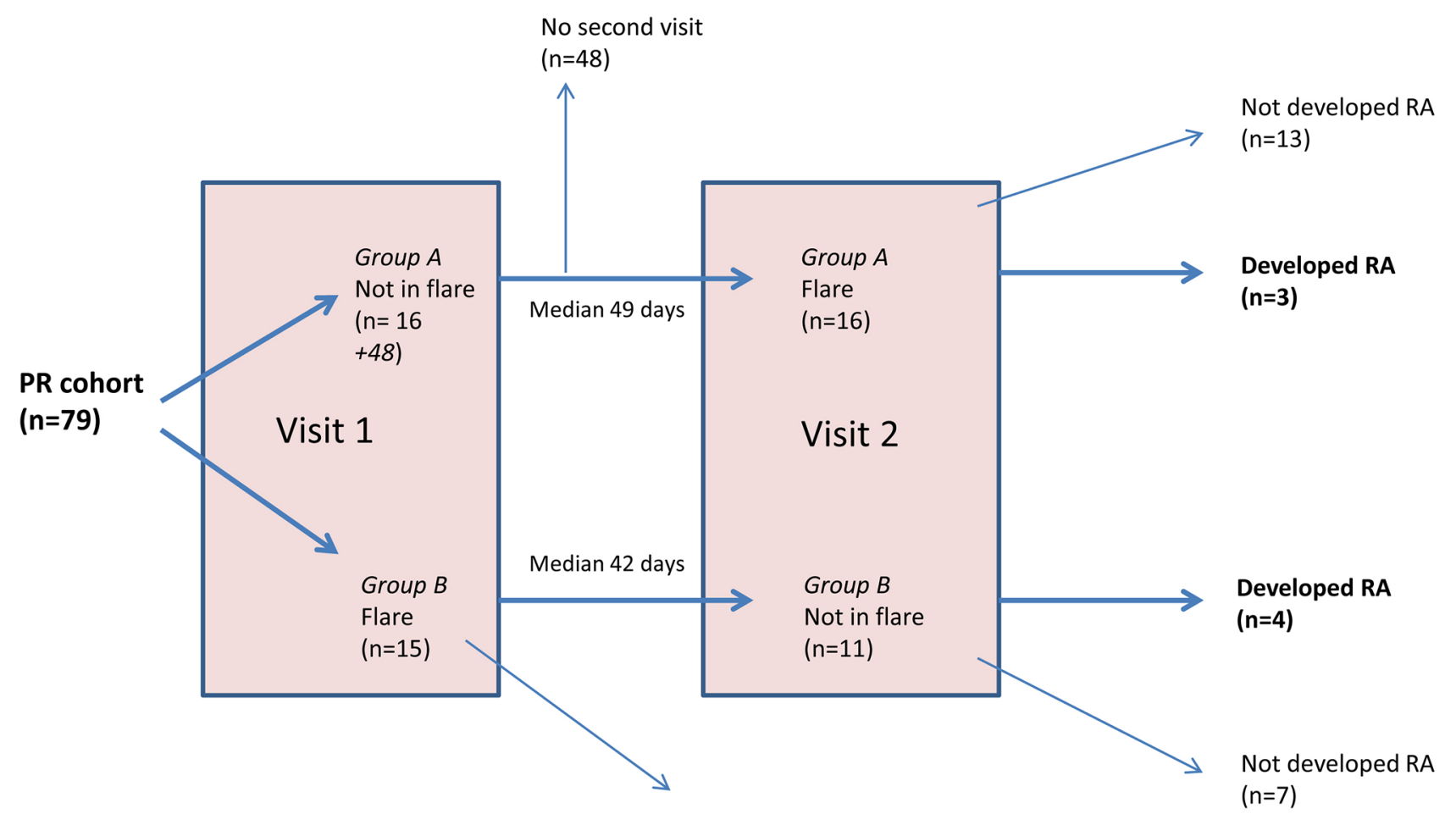

Not seen in non-flare

$(n=4)$

Figure 1 Flow chart showing patient visits for the palindromic rheumatism cohort. The cohort was followed according to patient-reported flares. Patients in Group A were not in flare at visit 1 and were in flare at visit 2. Patients in Group B were in flare at visit 1 but not at visit 2. Ultrasound assessments were performed at both visit 1 and visit 2. Ten out of $15(67 \%)$ patients with PR who were flaring at the initial visit had US abnormalities. Eleven of these patients subsequently attended a non-flare visit where only one (9\%) patient had US abnormalities. Of the patients who were not in flare at the initial visit and who subsequently attended for a flare visit, 9/16 (56\%) had US abnormalities. MRI assessments were performed during flare where possible. Patients were monitored for the development of persistent arthritis. PR, palindromic rheumatism; RA, rheumatoid arthritis; US, ultrasound.

\section{METHODS}

\section{Design}

A prospective analysis of a regional PR cohort was performed. For comparison, both a prospective and retrospective analysis of a cohort of CCP+ at-risk individuals and patients with NORA was also undertaken.

\section{Patients with PR}

Patients with PR were recruited from rheumatology clinics in Leeds and the Yorkshire region. Some patients with PR were also recruited through a national primary care programme adopted by the National Institute for Health Research Clinical Research Network. ${ }^{11}$

All patients were assessed at Chapel Allerton Hospital, Leeds, UK, and were recruited if the study rheumatologist diagnosed PR. In the absence of accepted classification criteria, PR was defined as 'a confirmed history or physical examination consistent with episodes of joint pain and swelling that returned to normal between episodes in the absence of an alternative diagnosis'.

Patients underwent clinical and US assessment at baseline and were followed according to patient-reported flares: those patients who were flaring at the initial visit were invited to reattend when they were not flaring; likewise, the patients who were not flaring at the initial visit were asked to telephone when they were having a flare and were seen within 48 hours. A flare episode was defined as two or more features of pain, swelling and erythema in or around at least one joint region that later normalised. Patients were divided into two subgroups according to the disease phase at the first assessment: patients in Group A were not in flare at their first assessment (ie, 'non-flare'), whereas patients in Group B were 'in flare' at their first assessment. For both groups, patients were re-evaluated at a second visit when the disease phase changed. US was performed at all flare and non-flare visits. MRI of the most affected region was performed during flare visits where possible. Patients were monitored for the development of persistent arthritis.

\section{Anti-CCP+ at-risk individuals}

$\mathrm{CCP}+$ at-risk individuals were recruited through a national primary care programme. ${ }^{11}$ This cohort has been previously described $^{12} 13$ and consists of subjects aged $>18$ years with non-specific MSK symptoms and a positive serum anti-CCP2 test but no clinical synovitis. Clinical and US assessments performed at the baseline visit in this cohort were included in the current analysis.

\section{Patients with NORA}

All patients with NORA met American College of Rheumatology (ACR)/European League Against Rheumatism (EULAR) 2010 classification criteria for RA. ${ }^{14}$ All patients with NORA were anti-CCP positive and disease-modifying antirheumatic drug (DMARD) naive at the time of assessment. Clinical and US assessments performed at RA diagnosis were included in the current analysis. 
Table 1 Patient characteristics

\begin{tabular}{|c|c|c|c|c|c|}
\hline & & $\operatorname{PR}(n=31)$ & & & \\
\hline & $\mathrm{CCP}+$ at risk $(\mathrm{n}=33)$ & During flare $(n=31)$ & During non-flare $(n=27)$ & NORA $(n=24)$ & $P$ values \\
\hline Age (years) ,mean (SD) & $47(15)$ & $49(14)$ & & $55(15)$ & 0.114 \\
\hline $\operatorname{Sex}(\% \mathrm{~F})$ & $88 \%(29 / 33)$ & $55 \%(17 / 31)$ & & $58 \%(14 / 24)$ & $0.01 * \dagger$ \\
\hline Anti-CCP positive (\%) & $100 \%(33 / 33)$ & $68 \%(21 / 31)$ & & $100 \%(24 / 24)$ & $<0.01$ \\
\hline RF positive (\%) & $18 \%(6 / 33)$ & $48 \%(15 / 31)$ & & $75 \%(18 / 24)$ & $<0.01 * \dagger$ \\
\hline DMARD naive (\%) & $100 \%(33 / 33)$ & $90 \%(28 / 31)$ & & $100 \%(24 / 24)$ & 0.06 \\
\hline Current smoker (\% yes) & $21 \%(7 / 33)$ & $39 \%(12 / 31)$ & & $29 \%(7 / 24)$ & 0.34 \\
\hline Never smoker (\% yes) & $58 \%(19 / 33)$ & $32 \%(10 / 31)$ & & $25 \%(6 / 24)$ & $0.03 \dagger$ \\
\hline Alcohol consumer (\% yes) & $42 \%(14 / 33)$ & $58 \%(18 / 31)$ & & $67 \%(16 / 24)$ & 0.18 \\
\hline FDR with RA (\% yes) & $24 \%(8 / 33)$ & $13 \%(4 / 31)$ & & $25 \%(6 / 24)$ & 0.43 \\
\hline Duration of symptoms (months) & $13(6,60)$ & $30(9,57)$ & $19(8.5,46)$ & $14(10,40)$ & 0.542 \\
\hline EMS (mins) & $0(0,30)$ & $90(0,120) n=29$ & $0(0,2.5)$ & $60(10,120)$ & $<0.01^{*} \dagger$ \\
\hline Symptoms in hands§ & $61 \%(20 / 33)$ & $84 \%(26 / 31)$ & $41 \%(11 / 27)$ & $100 \%(24 / 24)$ & $0.01 \dagger$ \\
\hline Symptoms in feet§ & $33 \%(11 / 33)$ & $26 \%(8 / 31)$ & $15 \%(4 / 27)$ & $58 \%(14 / 24)$ & 0.05 \\
\hline Symptoms in large joints§ & $64 \%(21 / 33)$ & $48 \%(15 / 31)$ & $44 \%(12 / 27)$ & $71 \%(17 / 24)$ & 0.23 \\
\hline Pain VAS (mm) & $23(4,50) n=31$ & $58(25,81) n=19$ & $11(3,34) n=21$ & $39(24,59) n=19$ & $0.03^{*}$ \\
\hline Fatigue VAS (mm) & $38(6.65) n=31$ & $42(22,64) n=19$ & $37(8,58) n=21$ & $42(23,60) n=19$ & 0.69 \\
\hline Global health VAS (mm) & $18(7,40) n=31$ & $41(16,55) n=19$ & $20(8,38) n=21$ & $29(16,50) n=21$ & 0.08 \\
\hline TJC 28 & $0(0,2)$ & $2(1,3)$ & $0(0,0)$ & $5(3,9)$ & $<0.01 * \dagger \ddagger$ \\
\hline TJC 53 & $1(0,2)$ & $1(1,2)$ & $0(0,1)$ & $5(3,7)$ & $<0.01 \dagger \ddagger$ \\
\hline SJC 28 & $0(0,0)$ & $1(1,2)$ & $0(0,0)$ & $2(1,6)$ & $<0.01 * \ddagger \dagger$ \\
\hline SJC 44 & $0(0,0)$ & $1(1,2)$ & $0(0,0)$ & $3(2,6)$ & $<0.01 * \dagger \ddagger$ \\
\hline $\mathrm{CRP}(\mathrm{mg} / \mathrm{dL})$ & $1.31(0.24,5.24) n=28$ & $9.9(1.1,26) n=29$ & $0(0,5.65) n=26$ & $6.5(0,9.38) n=23$ & $0.01^{*}$ \\
\hline DAS28CRP & $\mathrm{n} / \mathrm{a}$ & $\mathrm{n} / \mathrm{a}$ & $\mathrm{n} / \mathrm{a}$ & $3.48(3.18,4.56) n=21$ & $\mathrm{n} / \mathrm{a}$ \\
\hline DAS28CRP & $\mathrm{n} / \mathrm{a}$ & $\mathrm{n} / \mathrm{a}$ & $\mathrm{n} / \mathrm{a}$ & $3.48(3.18,4.56) n=21$ & $\mathrm{n} / \mathrm{a}$ \\
\hline
\end{tabular}

Baseline characteristics of PR patients seen in flare, CCP+ at-risk individuals and patients with NORA. Median and IQR are presented for scale variables. P values are given for comparisons between CCP+ at risk, PR flare and patients with NORA (Kruskall-Wallis and Fisher's exact tests). For significant results, pairwise tests were performed (MannWhitney $U$ test for scale variables).

${ }^{*} \mathrm{CCP}+$ vs PR flare $\mathrm{p}<0.05$.

${ }^{\dagger} \mathrm{CCP}+$ vs NORA $\mathrm{p}<0.05$.

${ }^{\ddagger} P R$ flare vs NORA $p<0.05$.

§Symptoms over the past week.

CCP+ at-risk, anti-cyclic citrullinated peptide positive at-risk individuals;CRP, C-reactive protein;DAS, disease activity score;DMARD, disease-modifying anti-rheumatic drug;EMS, early morning stiffness duration; FDR, first-degree relative; NORA, new-onset rheumatoid arthritis; PR, palindromic rheumatism; RF, rheumatoid factor; SJC, swollen joint count;TJC, tender joint count; VAS, visual analogue scale; n/a, not applicable.

Table 2 Ultrasound findings during and between flares of palindromic rheumatism

\begin{tabular}{|c|c|c|c|c|c|c|c|c|c|c|c|}
\hline & \multicolumn{4}{|c|}{ Synovial inflammation $\mathrm{n}(\%)$} & \multicolumn{4}{|c|}{ EC inflammation $\mathrm{n}(\%)$} & \multirow[b]{2}{*}{$\begin{array}{l}\text { Synovitis } \\
\text { only } n(\%)\end{array}$} & \multirow[b]{2}{*}{$\begin{array}{l}\mathrm{EC} \\
\text { inflammation } \\
\text { only } \mathrm{n}(\%)\end{array}$} & \multirow[b]{2}{*}{$\begin{array}{l}\text { Synovitis } \\
\text { and EC } \\
\text { inflammation } \\
\mathrm{n}(\%)\end{array}$} \\
\hline & $\begin{array}{l}\text { GS } \\
\text { synovitis } \\
(G S \geq 2)\end{array}$ & $\begin{array}{l}\text { PD } \\
\text { synovitis } \\
(P D \geq 1)\end{array}$ & Tenosynovitis & $\begin{array}{l}\text { Synovitis } \\
\text { (GS } \geq 1 \\
\text { and PD } \\
\geq 1 \text { ) }\end{array}$ & $\begin{array}{l}\text { Peritendinous } \\
\text { oedema }\end{array}$ & $\begin{array}{l}\text { Periarticular } \\
\text { inflammation }\end{array}$ & $\begin{array}{l}\text { Subcutaneous } \\
\text { oedema }\end{array}$ & $\begin{array}{l}\text { Any EC } \\
\text { inflammation }\end{array}$ & & & \\
\hline PR non-flare $(n=27)$ & $0(0)$ & $2(7)$ & $1(4)$ & $2(7)$ & $1(4)$ & $1(4)$ & $3(11)$ & $4(15)$ & $0(0)$ & $2(7)$ & $2(7)$ \\
\hline PR flare $(n=31)$ & $12(39)^{*}$ & $7(23)$ & $7(23)^{*}$ & $7(23)$ & $3(10)$ & $12(39)^{*}$ & $14(45)^{*}$ & $19(61)^{*}$ & $0(0)$ & $12(39)^{*}$ & $7(23)$ \\
\hline
\end{tabular}

Ultrasound findings at the clinically flaring site (ie hand, foot, shoulder) during flare and non-flare phases.

${ }^{*} \mathrm{p}<0.05$ (PR flare vs non-flare).

EC, extracapsular; GS, grey scale; PD, power doppler; PR, palindromic rheumatism.

\section{US evaluation}

US evaluation was performed by rheumatologists (RJW, MADA, $\mathrm{KM}, \mathrm{JLN}$ ) and sonographers (LH, KS) with extensive experience in MSK US who were blinded to patient group, symptoms and clinical assessment. All US examiners participated in a training session and agreed on the scanning protocol. A standardised 38-joint, 10-tendon US protocol was used at all visits (see online supplementary material 1 ). All available recorded images were scored by a single expert reader (MADA) who was blinded to all patient details, and this score was used in the analysis.
US scans were mainly performed using a General Electric (GE) Logiq E9 machine employing a $15-6 \mathrm{MHz}$ transducer. Copious gel was used as a standoff to avoid excessive transducer pressure. A small number of US scans were performed using a GE S7 machine. Power Doppler (PD) was assessed using a pulse repetition frequency set between 0.7 and $1.0 \mathrm{kHz}$, medium wall filter and gain adjusted until background noise was suppressed. Doppler frequency was $10 \mathrm{MHz}$.

Scoring of grey scale (GS) and PD synovitis was according to the EULAR Outcome Measures in Rheumatology (OMERACT) scoring system. ${ }^{15} 16$ Tenosynovitis was defined according to 
the OMERACT definition ${ }^{17}$ and scored as present or absent. To avoid overestimation, as scoring used for the analysis was based on central reading of images, synovitis was defined as $\mathrm{GS} \geq 1$ and $\mathrm{PD} \geq 1$.

Non-synovial EC abnormalities were frequently identified in our initial US assessments of PR flares and have previously been observed in patients with PR. ${ }^{18}$ Therefore, the following classification system for EC abnormalities was agreed by consensus (KM, MADA, RJW) after review of a randomised selection of flare images of different joint regions: periarticular inflammation: localised non-synovial soft tissue inflammation with or without PD signal outside the joint capsule and around the joint region; peritendinous oedema: oedema with or without PD signal occurring around a tendon without a tenosynovium; subcutaneous oedema: diffuse non-synovial soft tissue oedema with or without PD signal occurring outside the capsule and extending beyond the joint region. The figure 2 shows example images for each of these definitions. EC abnormalities were subsequently scored as present or absent.

\section{MRI evaluation}

MRI scanning was performed on the most symptomatic region during PR flare. Patients were scanned using a 3T Siemens Verio MRI scanner (Erlangen, Germany) (see online supplementary methods 1 ).

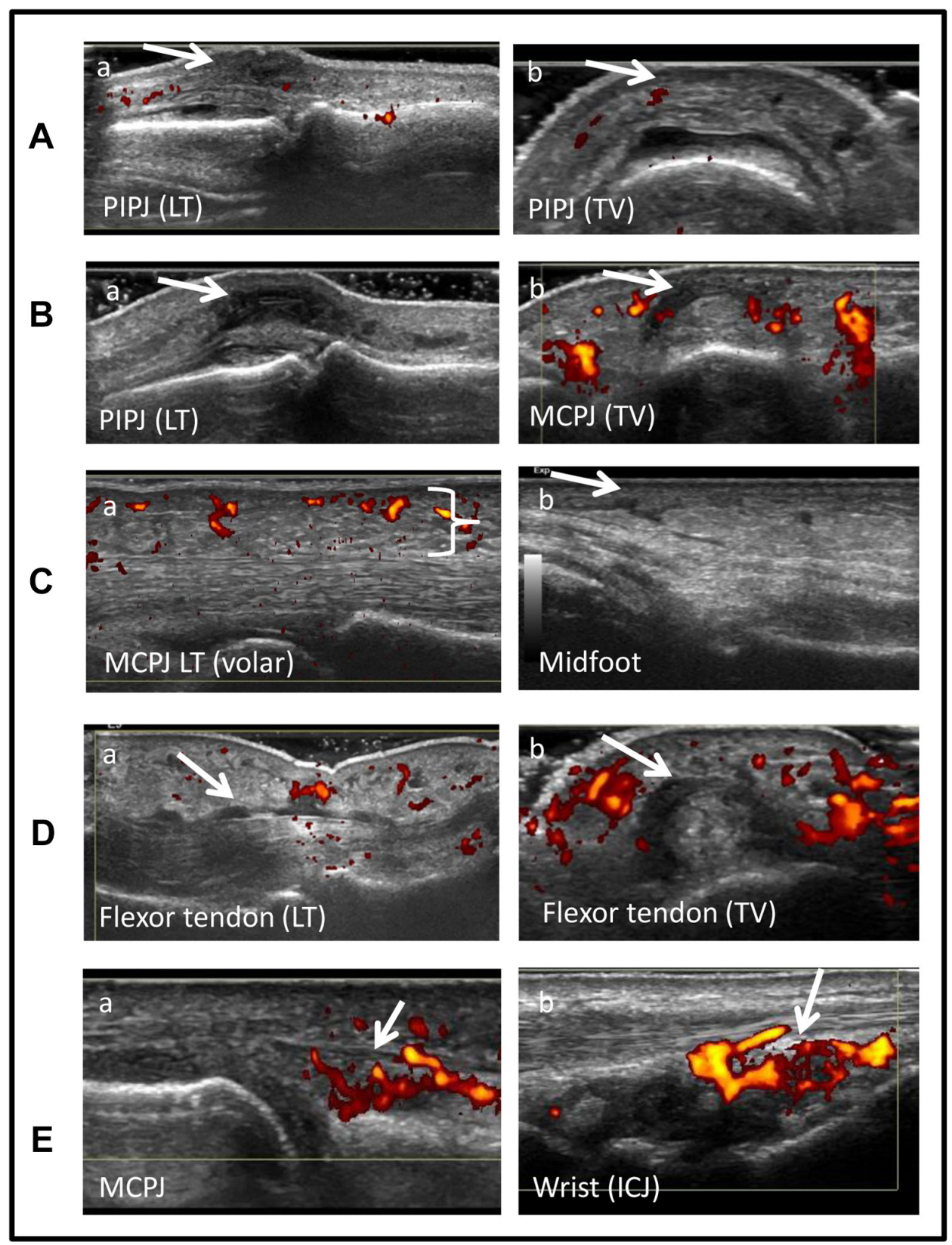

Figure 2 Ultrasound findings in flares of palindromic rheumatism. Representative images of the different types of ultrasound pathology detected at the flaring region are shown in the panels. (1) Peri-articular inflammation shown at a PIPJ in (a) LT and (b) TV. Joint effusion is also present. (2) Peri-tendinous oedema shown at (a) a PIPJ in LT and (b) a MCPJ in TV. (3) Subcutaneous oedema (indicated by the symbol "\}") shown at a MCPJ and midfoot. (4) Flexor tenosynovitis shown in (a) LT and (b) TV. Subcutaneous oedema is also present. (5) Synovitis shown at (a) MCPJ and (b) wrist ICJ. ICJ, intercarpal joint; LT, longitudinal; MCPJ, metacarpophalangeal joint; PIPJ, proximal interphalangeal joint; TV, transverse. 


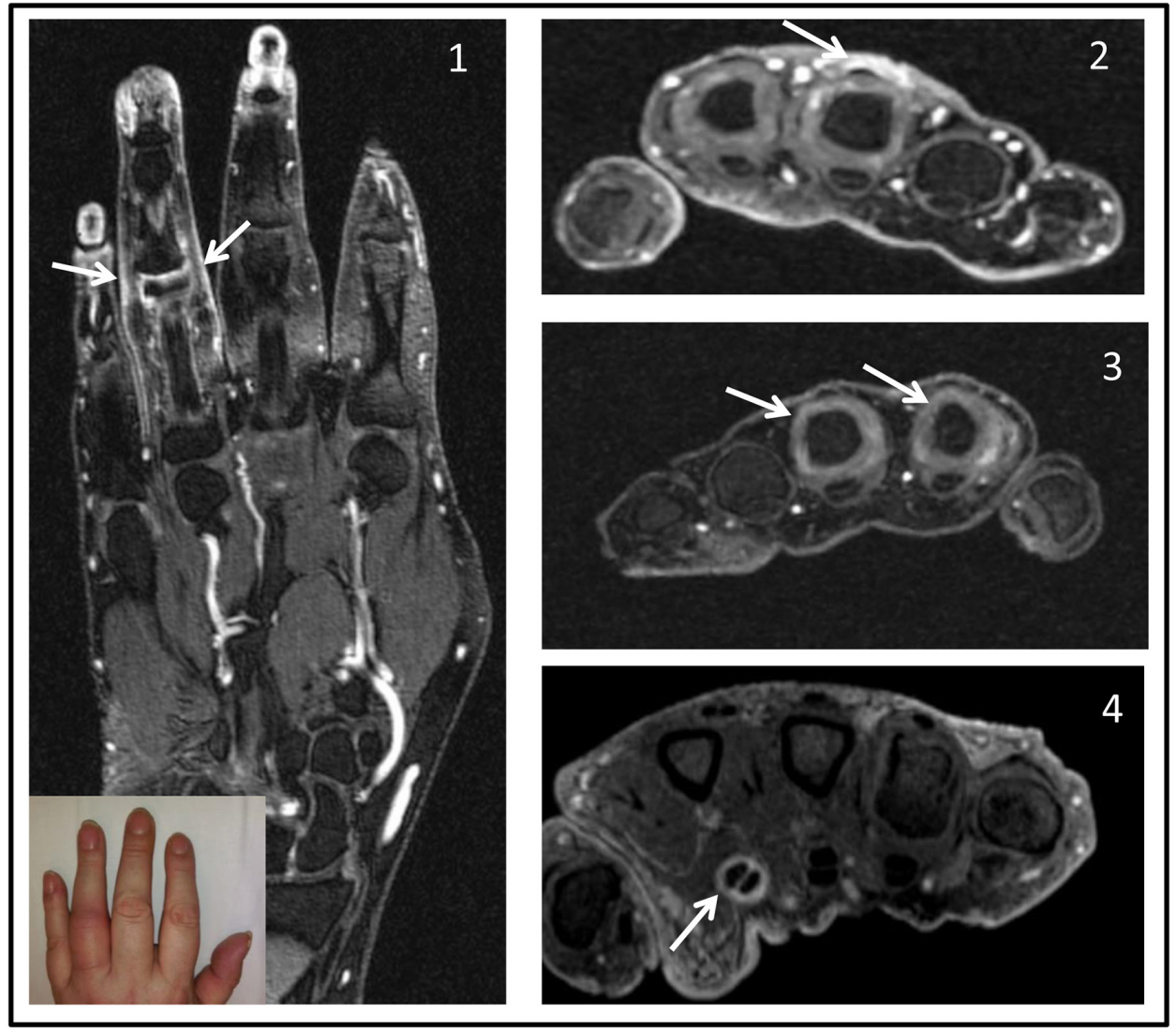

Figure 3 MRI findings in flares of palindromic rheumatism. Representative images of the different types of MRI pathology (highlighted by arrows) detected at the flaring region are shown in the panels. (1) Periarticular inflammation (shown for fourth PIPJ with clinical photograph insert). (2) Peritendinous oedema (shown for third extensor tendon at MCPJ level, the patient also has MCPJ synovitis). (3) Synovitis (shown for second and third MCPJs). (4) Tenosynovitis (shown for second flexor tendon). MCPJ, metacarpophalangeal joint; PIPJ, proximal interphalangeal joint.

All MRI scans were scored by an experienced reader (MADA) who was blinded to all patient and clinical details. The presence or absence of synovitis, bone marrow oedema (BME), tenosynovitis, erosions, peri-tendinous oedema and peri-articular inflammation was reported for the imaged region (ie, hand, knee, shoulder). Due to interference from coil artefacts, subcutaneous oedema was not included in this analysis. Synovitis and BME were reported according to the OMERACT RA MRI scoring system (RAMRIS). ${ }^{19}$ Tenosynovitis was defined according to the OMERACT MRI Tenosynovitis Scoring System ${ }^{20}$ and scored as present or absent. In the absence of an accepted definition for EC MRI abnormalities (ie, peri-articular inflammation and pertendinous oedema), these lesions were identified and scored using T1 fat-sat gadolinium enhanced sequences and reported descriptively. Periarticular inflammation was defined as EC effusion and/or postcontrast enhancement of the EC tissues on axial and coronal sequences over $\geq 3$ consecutive slices. Peritendinous oedema was defined as peritendinous effusion and/ or postcontrast enhancement outside the tendon sheath, seen on axial and coronal sequences over $\geq 3$ consecutive slices.

\section{Statistical analysis}

We tested the hypothesis that the frequency of synovial and EC US abnormalities during the flare episode would be different in patients with PR compared with anti-CCP+ at-risk individuals and patients with NORA. Therefore, the proportion of patients with PR with US abnormalities during a clinically defined flare in the hand(s)/wrist(s) was compared with the proportion of anti-CCP + at-risk individuals and patients with NORA with US abnormalities in the hands/wrists using $\chi^{2}$ or Fisher's exact test (where expected counts were $\leq 5$ cases). We also tested the hypothesis that the proportion of patients with PR with synovial and EC non-synovial US abnormalities would increase between non-flare and flare disease phases. Therefore, the proportion of patients with PR with US abnormalities in the clinically flaring region was compared in flare and non-flare disease phases using McNemar's exact test. Kruskall-Wallis and Fisher's exact tests were used to compare patient characteristics between groups. For significant results, pairwise tests were performed using Mann-Whitney $U$ test for scale variables.

\section{RESULTS}

\section{Patients}

Seventy-nine patients with PR met the study inclusion criteria and were recruited between May 2015 and May 2017. The cohort was followed prospectively according to patient-reported flares (figure 1). Fifteen out of 79 patients were flaring at the initial visit and 11 of these patients re-attended when they were not flaring. Sixteen out of 64 patients who were not flaring at their initial visit subsequently attended during a flare. In total, the $31 / 79$ patients who had an US assessment during a flare episode were included in the analysis. Seven out of $31(23 \%)$ patients developed persistent IA during the subsequent follow-up period; all these patients met the ACR/ 
Table 3 Ultrasound findings according to patient group

\begin{tabular}{|c|c|c|c|c|c|c|c|c|c|c|c|}
\hline & \multicolumn{4}{|c|}{ Synovial inflammation $\mathrm{n}(\%)$} & \multicolumn{4}{|c|}{ EC inflammation $\mathrm{n}(\%)$} & \multirow[b]{2}{*}{$\begin{array}{l}\text { Synovitis } \\
\text { only } n(\%)\end{array}$} & \multirow[b]{2}{*}{$\begin{array}{l}\mathrm{EC} \\
\text { inflammation } \\
\text { only } \mathrm{n}(\%)\end{array}$} & \multirow[b]{2}{*}{$\begin{array}{l}\text { Synovitis } \\
\text { and EC } \\
\text { inflammation } \\
\mathrm{n}(\%)\end{array}$} \\
\hline & $\begin{array}{l}\text { GS } \\
\text { synovitis } \\
(G S \geq 2)\end{array}$ & $\begin{array}{l}\text { PD } \\
\text { synovitis } \\
(P D \geq 1)\end{array}$ & Tenosynovitis & $\begin{array}{l}\text { Synovitis } \\
(G S \geq 1 \\
\text { and PD } \\
\geq 1)\end{array}$ & $\begin{array}{l}\text { Peri- } \\
\text { tendinous } \\
\text { oedema }\end{array}$ & $\begin{array}{l}\text { Peri - } \\
\text { articular } \\
\text { inflammation }\end{array}$ & $\begin{array}{l}\text { Subcutaneous } \\
\text { oedema }\end{array}$ & $\begin{array}{l}\text { Any EC } \\
\text { inflammation }\end{array}$ & & & \\
\hline $\begin{array}{l}C C P+\text { at risk } \\
(n=33)\end{array}$ & $10(32)$ & $4(13)$ & $4(13)$ & $4(12)$ & $0(0)$ & $1(3)^{*}$ & $1(3)^{*}$ & $2(6)^{*}$ & $4(12)$ & $2(6)^{*}$ & $0(0)^{*}$ \\
\hline PR flare $(n=26)$ & $9(35)$ & $6(23)$ & $7(27)$ & $6(23)$ & $2(8)$ & $12(46)$ & $13(50)$ & $17(65)$ & $0(0)$ & $11(42)$ & $6(23)$ \\
\hline Anti-CCP+ $(n=19)$ & $7(37)$ & $4(21)$ & $3(16)$ & $4(21)$ & $2(11)$ & $8(42)$ & $9(47)$ & $12(63)$ & $0(0)$ & $8(42)$ & $4(21)$ \\
\hline Anti-CCP- $\quad(n=7)$ & $2(29)$ & $2(29)$ & $4(57) \dagger$ & $2(29)$ & $0(0)$ & $4(57)$ & $4(57)$ & $5(71)$ & $0(0)$ & $3(43)$ & $2(29)$ \\
\hline NORA $(n=24)$ & $20(83) \ddagger$ & $17(71) \ddagger$ & $18(75) \ddagger$ & $17(71) \ddagger$ & $4(17)$ & $2(8) \ddagger$ & $5(21) \ddagger$ & $7(29) \ddagger$ & $11(46) \ddagger$ & $1(4) \ddagger$ & $6(25)$ \\
\hline
\end{tabular}

For comparative purposes only PR flares involving the hands/wrists are included.

${ }^{*} \mathrm{p}<0.05$ (PR flare vs $\mathrm{CCP}+$ at risk).

${ }_{\mathrm{p}}^{\mathrm{p}} \mathrm{p}=0.057$ (anti-CCP- vs anti-CCP+).

${ }_{\mathrm{f}} \mathrm{p}<0.05$ (PR flare vs NORA).

CCP+ at-risk, anticyclic citrullinated peptide antibody positive at-risk individuals; EC, extracapsular; GS, grey scale; NORA, new-onset rheumatoid arthritis; PD, power Doppler; PR, palindromic rheumatism.

EULAR 2010 classification criteria for RA. ${ }^{14}$ Of the complete cohort, $13 / 79$ (16\%) patients developed persistent IA; 47/79 patients were anti-CCP positive and of these 35 (74\%) did not develop persistent IA during the follow-up period.

Thirty-three CCP + at-risk individuals and 24 patients with NORA were included as control groups and were matched for age with patients with PR. Demographic and clinical characteristics are shown in table 1.

\section{Ultrasound findings in patients with PR}

US characteristics of patients with PR during flare (31 scans recorded) compared with US findings of the same region when the patient was not flaring (27 scans recorded) are shown in table 2 and online supplementary figure 1. US abnormalities were infrequently identified during non-flare and none had GS $\geq 2$ and $P D \geq 1$. Similarly, EC inflammation (ECI) was identified in only $4 / 27$ (15\%) of non-flare US scans. GS synovitis, tenosynovitis, periarticular inflammation and subcutaneous oedema were all less prevalent in non-flare scans compared with flare scans $(p<0.05)$. In contrast, there was no difference in the frequency of PD signal and peritendinous oedema between flare and non-flare US scans $(\mathrm{p}=0.289$ and $\mathrm{p}=0.625$, respectively). No erosions were identified on flare or non-flare scans.

Of the 27 patients who had non-flare scans, 11 were performed after the flare scan was captured. There was improvement in US abnormalities in all but one of these patients.

\section{Ultrasound findings during PR flare}

In the 31 patients in whom flares were captured, the flaring region was the hands/wrists in 26 patients, the foot/ankle in one patient, the knee in three patients and the shoulder in one patient.

A high frequency of ECI was seen (figures 2 and 3) during flare: in 19/31 (61\%) patients, one or more of periarticular inflammation, peritendinous oedema and/or subcutaneous oedema was identified. Interestingly in 12 patients, ECI was seen in the absence of GS (GS $\geq 2$ ) or PD synovitis. GS alone (GS $\geq 2$ ) was present in $12 / 31(39 \%)$ patients. Tenosynovitis and peritendinous oedema were detected in $7 / 31$ (23\%) and $3 / 31(10 \%)$ of patients, respectively. PD signal was present in only $7 / 31(23 \%)$ of patients. No differences in either synovial inflammation or ECI was found between in patients with PR according to anti-CCP status.

Five patients attended with more than one flare (online supplementary data). Overall, US inflammation did not appear to increase with sequential flares (online supplementary figure 4).
No patients had tophi, double contour sign, hyperechoic aggregates or any other US features suggestive of crystal arthritis.

\section{Comparison of PR with anti-CCP+ at-risk individuals and patients with NORA}

US abnormalities identified in patients with PR during flares involving the hands/wrists were compared with US abnormalities in the hands/wrists of anti-CCP+ at-risk individuals and patients with NORA (table 3 and online supplementary figures 2 and 3). PD signal was observed less frequently in patients with PR compared with patients with NORA $(p<0.05)$. In contrast, ECI was identified in the majority $(65 \%)$ of patients with PR but only $7 / 24(29 \%)$ of patients with NORA $(p=0.023)$. No patients with PR had synovitis on US of the flaring region without ECI also being present. Of note, the identification of ECI without synovitis at the flare site appeared to be specific for PR; $42 \%$ of patients with PR had this US phenotype but this occurred in only one pateient with NORA $(p=0.003)$ and two CCP+ at-risk individuals $(\mathrm{p}=0.0012)$.

\section{Comparison of MRI and ultrasound}

Eleven flares were captured by both MRI and US (in one patient two flares were captured by both imaging modalities). MRI appeared more sensitive than US for synovitis, tenosynovitis, peritendinous oedema and periarticular inflammation (online supplementary figure 5). Synovitis (taken as cut-off of RAMRIS >1) was identified in 7/11 (64\%) flares, whereas BME was reported in only one flare. Tenosynovitis and peritendinous oedema were identified by MRI in 5/11 (45\%) and $6 / 11(55 \%)$ flares, respectively. Periarticular inflammation was identified by MRI in 6/11 (55\%) flares. No MRI erosions were identified.

\section{Ultrasound features at progression to RA}

The US phenotype of seven patients with PR who developed RA during the follow-up period was similar to the patients with NORA who did not have a history of PR. US synovitis and/or tenosynovitis of the hands/wrists was present in 5/7 (71\%) of patients at progression to RA. In contrast, ECI was only present in $2 / 7(29 \%)$ of patients (online supplementary figure 3 ).

\section{DISCUSSION}

In the early stages of IA, identifying patients with persistent disease from those with a better prognosis can be difficult. While the presence of anti-CCP antibodies in patients with early synovitis is generally associated with poor prognosis, ${ }^{21} 22$ many 
patients with anti-CCP positive PR do not develop persistent IA. ${ }^{5}$ Indeed $74 \%$ of anti-CCP positive patients with PR in our cohort did not develop IA during follow-up. In clinical practice, these patients may be inappropriately treated (eg, with methotrexate) as they often meet ACR/EULAR criteria for RA. ${ }^{14}$

To our knowledge, this is the first study to demonstrate high-resolution imaging, especially US, may be used to distinguish PR from NORA at a single assessment. Isolated ECI appears to be specific for PR whereas synovitis and tenosynovitis is more frequently identified in NORA. This is important as PR carries a more favourable prognosis but often takes several assessments to diagnose clinically; we have identified a specific imaging phenotype which may facilitate earlier identification and therefore more appropriate management of these patients.

This is also the first study to use imaging to characterise ECI, synovitis and tenosynovitis in both flare and non-flare phases of PR. The high prevalence of peri-articular soft tissue inflammation and subcutaneous oedema on US during flare may explain clinical peri-arthritis in these patients. Tenosynovitis and peri-tendinous oedema, both identified on US and MRI, could also cause this. The specific US phenotype of ECI without synovitis suggests first that intra-articular inflammation may often not be responsible for the clinical features of PR flare and second that ECI may be mechanistically important rather than a secondary effect of an adjacent synovitis. This highlights the value of US in identifying the site of inflammation, particularly as most studies (including ours) have identified patients with PR clinically as having recurrent 'joint' swelling. Extra-articular abnormalities have been previously described in patients with PR who do not have US synovitis. ${ }^{18}$ However, contrary to our findings, a relatively high frequency of GS and PD synovitis has previously been reported. ${ }^{9} 101823$ Differences in patient characteristics may be one explanation. Our patients were comparatively early in their disease course (median 2.5 years) and all but three were DMARD- naive at the time of imaging. In contrast, patients in the other studies had experienced several years of disease $^{91023}$ and 45\%-61\% were on DMARD therapy at the time of assessment. ${ }^{923}$ It is possible that the phenotype we have described reflects de novo PR and this may change towards an RA phenotype with more prolonged disease duration and/or under the influence of immunomodulation.

The mechanism of ECI in PR is unclear and requires investigation; clinically, there are similarities with autoinflammatory diseases ${ }^{624}$ and the role of autoinflammation in PR is an important area for future research.

The low frequency of US abnormalities when patients were not flaring supports the notion that flares of PR are truly relapsing remitting and are important to distinguish from early IA. This is consistent with previous published data. ${ }^{23}$

The use of MRI is a strength of this study. In the majority of cases, MRI findings concurred with US findings as well as identifying additional abnormalities. Also, 2/11 patients had ECI on MRI in the absence of synovitis. The absence of erosions on MRI and identification of BME in only one patient confirms a distinct imaging pattern to early RA. Previous reports of MRI findings in PR flare are limited to a case report ${ }^{25}$ and a study of four patients in whom BME was identified in all cases and synovitis in three. ${ }^{10}$ Both studies describe a phenotype more akin to RA than we have observed.

Due to the transient and unpredictable nature of flares, it was not possible for the same US examiner to perform all scans. However, all sonographers were trained in the same centre and followed the same US protocol. In addition, all US and MRI scans were scored by an expert reader who was blinded to all clinical details. We acknowledge that the reliability of the proposed classification system for ECI should be assessed in future work; our findings should also be validated in other PR cohorts.

In conclusion, we identified a specific imaging phenotype in PR, which may be used to distinguish true PR from persistent IA in patients presenting with early arthritis. These findings may refine diagnosis and improve the management of this important condition.

Acknowledgements The authors would like to acknowledge Laura Horton and Kate Smith for performing ultrasound scans, Rob Evans and Brian Chaka for radiography support, and lan Weatherill and Philip Luxford for administrative support.

Contributors KM designed the study, collected and analysed the data and wrote the manuscript. MADA designed the study, scored the ultrasound and MRI images and helped write the manuscript. RJW helped design the ultrasound protocol and performed some of the ultrasound scans. JLN was one of the study clinicians and performed some of the ultrasound scans. WM helped with data analysis. AJG was responsible for MRI protocols. PE designed and led the study. All co-authors read and revised the manuscript.

Funding The study was supported by the National Institute for Health Research (NIHR) Leeds Clinical Research Facility. Additional support was provided by Arthritis Research UK (ARUK grant number 7174).

\section{Competing interests None declared.}

Patient consent Not required.

Ethics approval NHS Health Research Authority National Research Ethics Service Committee Yorkshire and the Humber, Leeds West.

Provenance and peer review Not commissioned; externally peer reviewed. Data sharing statement No additional data are available from this study.

\section{REFERENCES}

1 Quinn MA, Emery P. Window of opportunity in early rheumatoid arthritis: possibility of altering the disease process with early intervention. Clin Exp Rheumatol 2003;21(Suppl 31):S154-7.

2 Emad Y, Anbar A, Abo-Elyoun I, et al. In palindromic rheumatism, hand joint involvement and positive anti-CCP antibodies predict RA development after 1 year of follow-up. Clin Rheumatol 2014;33:791-7.

3 Russell AS, Devani A, Maksymowych WP. The role of anti-cyclic citrullinated peptide antibodies in predicting progression of palindromic rheumatism to rheumatoid arthritis. J Rheumatol 2006;33:1240-2.

4 Tamai M, Kawakami A, Iwamoto $\mathrm{N}$, et al. Contribution of anti-CCP antibodies, proximal interphalangeal joint involvement, HLA-DRB1 shared epitope, and PADI4 as risk factors for the development of rheumatoid arthritis in palindromic rheumatism. Scand J Rheumatol 2010;39:287-91.

5 Sanmartí R, Cabrera-Villalba S, Gómez-Puerta JA, et al. Palindromic rheumatism with positive anticitrullinated peptide/protein antibodies is not synonymous with rheumatoid arthritis. A longterm followup study. J Rheumatol 2012;39:1929-33.

6 Mankia K, Emery P. What can palindromic rheumatism tell us? Best Pract Res Clin Rheumatol 2017;31:90-8

7 Colebatch AN, Edwards CJ, Østergaard M, et al. EULAR recommendations for the use of imaging of the joints in the clinical management of rheumatoid arthritis. Ann Rheum Dis 2013;72:804-14.

8 D'Agostino MA, Terslev L, Wakefield R, et al. Novel algorithms for the pragmatic use of ultrasound in the management of patients with rheumatoid arthritis: from diagnosis to remission. Ann Rheum Dis 2016;75:1902-8.

9 Chen $\mathrm{HH}$, Lan JL, Hung GD, et al. Association of ultrasonographic findings of synovitis with anti-cyclic citrullinated peptide antibodies and rheumatoid factor in patients with palindromic rheumatism during active episodes. J Ultrasound Med 2009;28:1193-9.

10 Bugatti S, Caporali R, Manzo A, et al. Ultrasonographic and MRI characterisation of the palindromic phase of rheumatoid arthritis. Ann Rheum Dis 2012:71:625-6.

11 Nam JL, Hunt L, Hensor EM, et al. Enriching case selection for imminent RA: the use of anti-CCP antibodies in individuals with new non-specific musculoskeletal symptoms a cohort study. Ann Rheum Dis 2016;75:1452-6.

12 Rakieh C, Nam JL, Hunt L, et al. Predicting the development of clinical arthritis in antiCCP positive individuals with non-specific musculoskeletal symptoms: a prospective observational cohort study. Ann Rheum Dis 2015;74:1659-66.

13 Nam JL, Hensor EM, Hunt L, et al. Ultrasound findings predict progression to inflammatory arthritis in anti-CCP antibody-positive patients without clinical synovitis. Ann Rheum Dis 2016;75:2060-7.

14 Aletaha D, Neogi T, Silman AJ, et al. 2010 Rheumatoid arthritis classification criteria: an american college of rheumatology/European League Against Rheumatism collaborative initiative. Arthritis Rheum 2010;62:2569-81. 
15 D'Agostino MA, Terslev L, Aegerter P, et al. Scoring ultrasound synovitis in rheumatoid arthritis: a EULAR-OMERACT ultrasound taskforce-Part 1: definition and development of a standardised, consensus-based scoring system. RMD Open 2017;3:e000428.

16 Terslev L, Naredo E, Aegerter P, et al. Scoring ultrasound synovitis in rheumatoid arthritis: a eular-omeract ultrasound taskforce-Part 2: reliability and application to multiple joints of a standardised consensus-based scoring system. RMD Open 2017;3:e000427.

17 Naredo E, D'Agostino MA, Wakefield RJ, et al. Reliability of a consensus-based ultrasound score for tenosynovitis in rheumatoid arthritis. Ann Rheum Dis 2013;72:1328-34

18 Chen H-H, Chen D-Y, Hsieh T-Y, Chen HCD, Hung G, et al. Predicting the progression of palindromic rheumatism to rheumatoid arthritis: the role of ultrasonography and anti-cyclic citrullinated peptide antibodies. J Med Ultrasound 2010;18:17-26.

19 Østergaard M, Peterfy C, Conaghan P, et al. Omeract rheumatoid arthritis magnetic resonance imaging studies. Core set of MRI acquisitions, joint pathology definitions, and the OMERACT RA-MRI scoring system. J Rheumatol 2003;30:1385-6
20 Glinatsi D, Bird P, Gandjbakhch F. Development and validation of the OMERACT rheumatoid arthritis magnetic resonance tenosynovitis scoring system in a multireader exercise. J Rheumatol 2017;44:1688-93.

21 Freeston JE, Wakefield RJ, Conaghan PG, et al. A diagnostic algorithm for persistence of very early inflammatory arthritis: the utility of power Doppler ultrasound when added to conventional assessment tools. Ann Rheum Dis 2010;69:417-9.

22 Raza K, Breese M, Nightingale P, et al. Predictive value of antibodies to cyclic citrullinated peptide in patients with very early inflammatory arthritis. J Rheumatol 2005;32:231-8.

23 Cabrera-Villalba S, Ramirez J, Salvador G, et al. Is there subclinical synovitis in patients with palindromic rheumatism in the intercritical period? a clinical and ultrasonographic study according to anticitrullinated protein antibody status. J Rheumatol 2014:41:1650-5.

24 Cañete JD, Arostegui Jl, Queiró R, et al. An unexpectedly high frequency of MEFV mutations in patients with anti-citrullinated protein antibody-negative palindromic rheumatism. Arthritis Rheum 2007;56:2784-8.

25 Ueda S, Horino T, Arii K, et al. Magnetic resonance imaging of palindromic rheumatism. Rheumatol Int 2008;29:87-9. 